\title{
Interactive comment on "Shell dissolution observed in Limacina helicina antarctica from the Ross Sea, Antarctica: paired shell characteristics and in situ seawater chemistry" by Kevin M. Johnson et al.
}

\section{Anonymous Referee \#2}

Received and published: 23 January 2017

There is a growing body of papers linking carbonate chemistry and shell dissolution, firmly establishing the link between the carbonate chemistry and shell dissolution. While this manuscript only reestablishes this correlation, it does not contribute much to the development of new knowledge or approaches. Contrary of what the authors are claiming this being the first study to combine natural variability and shell dissolution, that is not the case, not it is presented as such. There are several issues with this manuscript that need to be consistently addressed. Firstly, the major drawback of the paper is the lack of the working hypotheses. That leaves the work more a compilation of different facts, without any proper integration. Authors have to reconsider how to bring 
the results in the framework that addresses NEW questions and form the hypothesis around.

Secondly, this is a poorly designed and conceived study, with inconsistent methodological and statistical approaches that cannot be verified. The authors have obviously used a bit different approach in preparing the samples that is different from previous approaches. What was the reason for doing this? Was a method systematically tested and how was this done for the authors to be confident in the interpretation of their results? Did the periostracum stay intact or could this method have caused breaching of the periostracum at any stage or are the authors confident that no additional damaged were produced during the process? I would urge the authors to prepare and present additional results on pteropods that did not undergo this treatment but rather standardized treatment.

The authors did not have any controls in this study - I request for all the controls to be consistently presented in the paper.

I am perplexed on why the temperature of the water was not measured along with $\mathrm{pH}$. Using temperature from the multi-year series for calculating carbonate chemistry parameters can have major drawbacks and error propagations. Given that temperature can significantly impact aragonite saturation state, it is an imperative to include standard error and uncertainty range in all carbonate chemistry parameters throughout the whole season of measured $\mathrm{pH}$.

In regards to dissolution estimates, I cannot trace the tows to the carbonate chemistry conditions. Make that link more explicit. I wonder how the authors explain excessive presence of Type III dissolution when the organisms have been exposure to supersaturated conditions throughout the whole study. Is it possible that the damages actually originated before the samples were collected - during the austral winter? If that was the case then the carbonate chemistry presented does not really matter.

Printer-friendly version

Where are the figures from Figure 6 from and when where they collected? Indicate 
how many individuals per each tow look like 6a-c, d-f, g-j. Is image g-j all from one organism?

Please, clarify what additional information is captured in Figure 8 that has not been captured in Figure 7. If no different, remove Figure 8.

The authors have decided to analyze only the first whorl - why is that? I disagree with the authors that analyzing such a small surface can be unbiased and representative. While this is the easiest approach it does not give the full insight into dissolution. I would like to see the whole shell surface analyzed instead and presented. Only in such way, the results of this study can be comparable to the other studies.

The authors need to provide more discussion about the periostracum. I do not see any evidence that periostracum would be mechanically or chemically damaged - does this then mean that the dissolution is a results of carbonate chemistry conditions, or could it be breached during the process? I think this is an ideal study where the authors could potentially discuss their results in the light of the recent arguments on what is causing dissolution - e.g. Peck et al. (2016) stating that this only happens under the breach of the periostracum, while other authors claimed that dissolution is not indiscriminately linked to breached periostracum, occurring sporadically throughout the shell. I would encourage the authors to search for the evidence that can potentially bring more insights into divided views and present an extended discussion on this.

The results in Figure 7 are not consistently analyzed to include all possible comparisons within and between the tows. What is missing is the variability per each tow, and comparison between ALL the tows (not just in the selected few as done now). Once all the additional analyses have been performed, the authors need to discuss the interand intra-sample variability in dissolution in much more details - there is obvious a lot of variability on the individual level - why is that? Also, there is not much variability on the carbonate chemistry level, yet quite a lot of difference in shell dissolution between Nov and Dec sampling - provide an explanation for this. Is this due to cumulative expo-

Printer-friendly version

Discussion paper 
sure? Is dissolution function of size of the animal? In the light of examining dissolution through the exposure point of view, I want to see dissolution as a function of cumulative exposure, not just of in situ omega.

One poorly integrated aspect of this study are shell measurements. How many individuals were analyzed for each bar in Figure 4? I would like to see correlation between shell dissolution and size - per time and per each tow. I suggest the size of each individual with dissolution to be presented in Figure 7. This is the way to demonstrate if/how the size of the organism matters and it can also potentially show if the damages have occurred in the period before sampling. Discuss the implication of these results! Also, correlated shell size to variability in carbonate chemistry conditions $(\mathrm{pH}$, omega) and most importantly, how does cumulative exposure impact the size? These are all the questions that this study can potentially address but have so far failed to do so.

As calculated, density calculations only refer to the density of the organism in the subsample. I would like to see abundances calculated instead $(\mathrm{m}-2)$ as it makes it comparable with other studies. As above with shell dissolution, make density correlation with carbonate chemistry (omega, $\mathrm{pH}$ ) and shell size.

The discussion is poorly written. Instead of discussing their own results, the authors are presenting the results of the other studies -this belongs to the introduction. The discussion needs to be completely restructured and more cohesive.

In the discussion, the authors are referring to the experimental results ...Laboratory experiments conducted on this same research project showed that holding Limacina in high pCO2 (900 $\mu \mathrm{atm})$ for 14 days resulted in significant shell dissolution. 'Where is this statement coming from and how come the authors do not choose to present the results? The only way that the results from natural environment are confirmed is by experimental study, and for this, the results need to be clearly presented!

Also, explain this statement: Our own studies of juvenile pteropods found that $L$. $h$. antarctica exhibited a differential response to pCO2 only under ambient temperatures

Printer-friendly version

Discussion paper
Interactive comment 
$\left(-1.0^{\circ} \mathrm{C}\right)$ What sort of differential responses are considered? Explain and corroborate!

Interactive comment on Biogeosciences Discuss., doi:10.5194/bg-2016-467, 2016. 